\title{
Cinética de secagem e avaliação físico-química de fruta-pão (Artocarpus altilis) variedade seminífera
}

\author{
Kalline Kelly Oliveira Resende ${ }^{1}$, Sumária Sousa e Silva1, Sumaya Ferreira Guedes ${ }^{1}$, Raquel \\ Aparecida Loss ${ }^{1}$
}

${ }^{1}$ Universidade do Estado de Mato Grosso, Campus Deputado Estadual Renê Barbour, Barra do Bugres, Mato Grosso, Brasil. E-mail: kalline-kelly@hotmail.com, sumariasousa@gmail.com,su_sumaya@yahoo.com.br, raquelloss@unemat.br

Recebido: 15/05/2018; Aceito: 17/12/2018.

\begin{abstract}
RESUMO
O presente trabalho teve como objetivo realizar a secagem da fruta-pão variedade Seminífera e avaliar os parâmetros físico-químicos da fruta in natura e desidratada (farinha). As frutas foram separadas em partes (polpa, semente, caroço e casca) e submetidas às análises de umidade, $\mathrm{pH}$, acidez, cinzas, e também foi determinado o teor de amido. Para realização da cinética de secagem, utilizou-se estufa com circulação forçada de ar e os dados foram analisados com modelos matemáticos. Os resultados mostraram que na fruta in natura o maior teor de umidade e cinzas foi encontrado no caroço, com $89 \%$ e $9,33 \%$, respectivamente. Já na forma de farinha, a parte que apresentou o maior teor de umidade e cinzas foi a polpa. No geral, a fruta apresentou $\mathrm{pH}$ ácido, tanto in natura como desidratada, e menor acidez na fruta in natura quando comparada com farinha. A parte da fruta que apresentou o teor de amido mais elevado foi a semente (38\%), seguida da polpa $(19,10 \%)$, do caroço $(15,94 \%)$ e da casca $(15,94 \%)$. Com relação à cinética de secagem, os dados mostram que o modelo de Page foi o que melhor se ajustou aos dados experimentais, uma vez que apresentou menor erro e maior correlação. Dessa forma, concluise que é viável a produção de farinha a partir da fruta-pão de caroço, que, por apresentar boa avaliação físicoquímica e elevada quantidade de amido, pode ser uma fonte alternativa em substituição às farinhas tradicionais.
\end{abstract}

Palavras-chave: Amido, Farinha, Fruta exótica.

\section{Kinetics of drying and physicochemical evaluation of breadfruit (Artocarpus altilis) seminifer variety}

\begin{abstract}
The present work had the objective of drying the breadfruit Seminifer variety, and evaluation of the physicochemical parameters of the fruit in natura and dehydrated (flour). The fruits were separated into parts (pulp, seed, core and shell) and subjected to analysis of moisture, $\mathrm{pH}$, acidity, ashes, and starch quantification. To perform the kinetics, a drying oven with forced air circulation was used and the data were analyzed with mathematical models. The results showed that in the in natura fruit the highest content of moisture and ashes was found in the core, with $89 \%$ and $9.33 \%$, respectively. In the form of flour, the part that presented the highest moisture content and ashes was the pulp. In general, the fruit had acid pH, both in natura and dehydrated, and less acidity in the fruit in natura when compared to flour. The proportion of fruit with the highest starch content was seed (38\%), followed by pulp (19.10\%), core (15.94\%) and shell (15.94\%). Regarding drying kinetics, the data show that the Page model was the one that best fit the experimental data, since it presented smaller error and greater correlation. In this way, it is concluded that it is viable to produce flour from the breadfruit. Thus, due to the good physicalchemical evaluation and high amount of starch can be an alternative source to replace traditional flours.
\end{abstract}

Keywords: Starch, Flour, Exotic fruit. 


\section{Introdução}

O Brasil possui grande variedade de frutíferas nativas com importante potencial gastronômico e tecnológico. Entretanto, parte da biodiversidade brasileira de frutas é desconhecida pela população devido à substituição das variedades nativas por outras de maior apelo comercial. Dentre essas frutas, destacam-se abiu (Pouteria caimito) (Almeida et al., 2008), araçá-boi (Eugenia stipitata) (Virgolin et al., 2017), cajá (Spondias lutea), cupuaçu (Theobroma grandiflorum), murici (Byrsonima crassifolia) (Carlos et al., 2017), noni (Morinda citrifolia Linn) (Farias et al., 2018), bacuri (Platonia insignis) (Canuto et al., 2010), e fruta-pão (Artocarpus altilis) (Luciano et al., 2017, 2018). Esta última desperta a atenção de estudiosos, não somente pela sua crescente produtividade, mas também por conter alto teor de carboidratos, vitaminas $\left(\mathrm{B}_{1}, \mathrm{~B}_{2} \mathrm{e}\right.$ C), cálcio, fósforo e ferro. Essas propriedades interessantes podem trazer benefícios à saúde pelo uso medicinal popular de diferentes partes da planta (folhas, frutos, látex, sementes, raízes e casca), ainda pouco exploradas (Pradhan et al., 2012; Souza e Souza, 2012).

A fruta-pão é originária da região indomalásia (Ilhas de Java e Sumatra) e pertence à família Moraceae, sendo cultivada em todas as ilhas do arquipélago asiático e nas regiões tropicais de todo o mundo. Foi introduzida no Brasil, especificamente no estado do Pará, originando assim a sua dispersão pelo Brasil (Luciano et al., 2017). Atualmente, pode ser encontrada nos estados do Pará, São Paulo, Rio de Janeiro, Bahia, Paraíba, Alagoas, Sergipe, Pernambuco, Ceará e Mato Grosso. Ela adapta-se bem ao clima de baixa altitude, com pluviosidade acima de $1.200 \mathrm{~mm}$ bem distribuída durante o ano, temperatura média de $25{ }^{\circ} \mathrm{C}$, sem ocorrência de geadas e umidade relativa acima de $70 \%$. Nessas condições edafoclimáticas, as árvores adultas podem produzir anualmente $100 \mathrm{~kg}$ ou mais de frutos por safra, com peso médio de 1,0 a $1,5 \mathrm{~kg}$. A produção média no Brasil é de $48 \mathrm{~kg}$ por planta/safra (Sacramento et al., 2009).

São conhecidas duas variedades de fruta-pão: a Apyrena, conhecida por fruta-pão de massa, que não possui sementes, e a Seminífera, conhecida por frutapão de caroço, que apresenta numerosas sementes. A variedade Seminífera diferencia-se por apresentar no epicarpo inúmeras "espículas", cujo aspecto interno é muito semelhante ao fruto da jaqueira (Artocapus heterophyllus Lam.) (Madruga et al., 2014). A parte comestível do fruto é representada pelas sementes, que podem variar de 50 a 60 unidades por fruto. Geralmente, são consumidas após a cocção, tendo sabor semelhante ao da castanha-portuguesa (Castanea sativa Miller) (Sacramento et al., 2009).

A fruta-pão da variedade Seminífera é considerada uma planta versátil, devido ao aproveitamento de todas as suas partes, desde a raiz até o látex, sendo aproveitada para alimentos, medicina popular e essências (Souza e Souza, 2012). As plantas caracterizam-se por apresentar um porte mais elevado do que a variedade Apyrena. Além disso, apresentam rápido crescimento, podendo alcançar uma altura de até $30 \mathrm{~m}$, com mais de $1,5 \mathrm{~m}$ de diâmetro (Sacramento et al., 2009).

Independente das informações promissoras sobre a fruta, em muitas partes do mundo ela continua sendo uma espécie subutilizada. As maiores dificuldades de se utilizá-la são: complicações na propagação, sazonalidade do fruto, possuindo uma vida útil muito pequena, desafios com a inserção de um novo alimento na culinária tradicional e a falta de informações dessa espécie. Dessa forma, uma alternativa seria usá-la como matéria-prima para produção de farinha, uma vez que a fruta-pão é rica em carboidratos, podendo, por exemplo, ser utilizada como farinha instantânea para a fabricação de purês, mingaus, suflês, entre outros. Assim, além de agregar valor, favorece a renda dos pequenos agricultores que investem nessa fruta exótica e regional (Souza e Souza, 2012). Diante do exposto, a caracterização físico-química da fruta é de suma importância para ser aproveitada em formulações alimentícias.

De acordo com Melo et al. (2013), a secagem é um processo tradicional na preservação de alimentos, que diminui a disponibilidade da água (atividade de água) para reações de deterioração, aumenta a estabilidade e reduz o volume. Celestino (2010) afirma que a secagem é a operação unitária por meio da qual um líquido é removido de um material, possibilitando algumas vantagens como: prolongamento da vida de prateleira do produto, redução significativa das perdas dos alimentos pós-colheita e fácil comercialização e transporte dos produtos. Além disso, os dados contidos nas curvas de secagem são essenciais para o andamento de processos e para a avaliação de equipamentos, sendo possível determinar pelas curvas dados tais como o tempo que pode durar a produção, o tempo gasto para secar uma quantidade qualquer de produto, estimar também o gasto energético que recairá sobre o preço final do processamento, podendo assim influenciar no preço final dos produtos. Assim, combina a economia de volume e massa no transporte e no armazenamento em razão da compactação e da leveza, com os efeitos benéficos da estabilidade microbiológica e química, permitindo que o produto tenha maior durabilidade (Celestino, 2010; Melo et al., 2013).

Neste contexto, o objetivo deste trabalho foi realizar a avaliação físico-química de diferentes partes da frutapão variedade Seminífera (polpa, semente, caroço e casca), in natura e desidratada (farinha), e avaliar as curvas de secagem das farinhas obtidas. 


\section{Material e Métodos}

\section{Local e preparo da matéria-prima}

O presente trabalho foi desenvolvido no Laboratório de Química da Universidade do Estado de Mato Grosso - UNEMAT, campus Universitário Deputado Estadual Renê Barbour. As frutas foram adquiridas em uma fazenda localizada na cidade de Barra do Bugres-MT, geograficamente situada a $15^{\circ} 04^{\prime} 21^{\prime \prime}$ Sul e a $57^{\circ} 10^{\prime} 52^{\prime \prime}$ Oeste, a 150 km de distância da capital Cuiabá.

As frutas foram colhidas maduras no segundo semestre de 2016. Após colheita, foram higienizadas com detergente comercial e lavadas em água corrente. Após a limpeza, foram cortadas ao meio e subdivididas em casca, sementes, polpa e caroço, sendo que cada uma das partes foi triturada separadamente em liquidificador doméstico e armazenadas em freezer a $-18{ }^{\circ} \mathrm{C}$ até o momento das análises físico-químicas de acidez, pH, cinzas e umidade. Após a caracterização físico-química da fruta in natura, as amostras das diferentes partes do fruto foram submetidas à secagem em estufa com circulação de ar forçada a $40{ }^{\circ} \mathrm{C}$. As amostras secas foram trituradas para obtenção das farinhas, as quais foram submetidas às mesmas análises físico-químicas das amostras in natura. Além disso, as farinhas foram analisadas em relação ao teor de amido.

\section{Curvas de secagem}

Para a obtenção das curvas de secagem das diferentes partes da fruta-pão em placas Petri previamente secas, foram pesados $5 \mathrm{~g}$ de amostras e incubadas em estufa com circulação de ar forçada a $40{ }^{\circ} \mathrm{C}$, sendo realizadas pesagens periódicas até peso constante. As massas secas obtidas ao longo das pesagens foram convertidas em razão de umidade (RU), pela Equação 1. A curva de secagem foi realizada em triplicata

$$
R U=\frac{(U-U \theta)}{\left(U i-U_{\theta}\right)}
$$

onde: $\mathrm{U}=$ teor de água do produto, decimal b.s; $\mathrm{Ui}=$ teor de água inicial do produto, decimal b.s; $\mathrm{Ue}=$ teor de água de equilíbrio do produto, decimal b.s.

$\mathrm{O}$ tratamento dos dados experimentais das curvas de secagem foi realizado em função da aplicação de três modelos matemáticos empíricos (Tabela 1). A escolha do melhor modelo que se ajustou aos dados experimentais foi em função da correlação e do erro padrão médio.

\section{Caracterização físico-química}

Foram empregados os métodos descritos pelo Instituto Adolfo Lutz (2008) para a avaliação físicoquímica da fruta-pão. Os parâmetros avaliados foram: acidez em ácido cítrico (\%), pH, cinzas (\%), umidade $(\%)$ e amido (\%) das diferentes partes do fruto (polpa, semente, caroço e casca).

\section{Umidade}

Para a determinação da umidade, em um cadinho previamente tarado, foram pesados em balança semianalítica com precisão de 0,001g (Shimadzu Corporation, modelo UX420H, Brasil), $5 \mathrm{~g}$ da amostra e levadas em estufa (DeLeo Equipamentos Laboratoriais, modelo DL-SED, Brasil) para secagem direta a $105^{\circ} \mathrm{C}$ até peso constante (Instituto Adolf Lutz, 2008).

\section{Acidez,}

Para a determinação da acidez, foram pesados $3 \mathrm{~g}$ de amostra em um erlenmeyer, em balança semi-analítica (Shimadzu Corporation, modelo UX420H, Brasil), adicionados $50 \mathrm{~mL}$ de água destilada e 3 gotas do indicador (fenolftaleína). A solução foi titulada com hidróxido de sódio $0,1 \mathrm{~mol} \mathrm{~L}^{-1}$, previamente padronizado ( $\mathrm{fc}=1,036304087$ ) até coloração rósea (Instituto Adolf Lutz, 2008).

\section{pH}

$\mathrm{Na}$ determinação do $\mathrm{pH}, 5 \mathrm{~g}$ da amostra foram diluídos em $50 \mathrm{~mL}$ de água destilada e então submetidos à leitura de $\mathrm{pH}$ em potenciômetro previamente calibrado, com precisão de $\pm 0,01$ (Marconi Equipamentos para Laboratório LTDA, modelo RS232, Brasil) (Instituto Adolf Lutz, 2008).

\section{Cinzas}

Foram pesados em balança semi-analítica (Shimadzu Corporation, modelo UX420H, Brasil) $5 \mathrm{~g}$ de amostra, em um cadinho previamente tarado, sendo a amostra carbonizada e, em seguida, incinerada em mufla (Quimis Aparelhos Científicos LTDA, modelo Q$318 \mathrm{M} 21$, Brasil) a uma temperatura de $550{ }^{\circ} \mathrm{C}$ até que apresentasse coloração branca ou levemente acinzentada, não contendo pontos negros. Em seguida, o cadinho foi transferido para o dessecador por 30 minutos, sendo então pesados (Instituto Adolf Lutz, 2008).

Tabela 1. Modelos matemáticos para a representação da cinética de secagem.

\begin{tabular}{cc}
\hline Modelos matemáticos & Equações \\
\hline Page & $\mathrm{RU}=\exp \left(-\mathrm{k} * \mathrm{t}^{\mathrm{n}}\right)$ \\
Henderson e Pabis & $\mathrm{RU}=\mathrm{a} \exp (-\mathrm{k} * \mathrm{t})$ \\
Midilli e Kucuk & $\mathrm{RU}=\mathrm{a} \exp \left(-\mathrm{k} * \mathrm{t}^{\mathrm{n}}\right) \mathrm{b} * \mathrm{t}$ \\
\hline
\end{tabular}

$\mathrm{RU}=$ razão do teor de água do produto, adimensional; $\mathrm{t}=$ tempo de secagem, em $\mathrm{h} ; \mathrm{k}=$ coeficiente de secagem, em $\mathrm{s} 1 ; \mathrm{a}, \mathrm{b}, \mathrm{n}$ e $\mathrm{k}=\mathrm{constante}$ do modelo, adimensional. 


\section{Amido}

A determinação do amido foi realizada de acordo com o método 043/IV descrito pelo Instituto Adolfo Lutz (2008), onde foram pesados $5 \mathrm{~g}$ de amostra em tubo falcon de $50 \mathrm{~mL}$ adicionados $20 \mathrm{~mL}$ de éter de petróleo e agitados em vortex por 5 minutos. Posteriormente, a solução foi transferida para um erlenmeyer de $500 \mathrm{~mL}$ juntamente com $100 \mathrm{~mL}$ de álcool etílico $70 \%$. Em seguida, a mistura foi aquecida a $100{ }^{\circ} \mathrm{C}$ por 1 hora, utilizando um pequeno funil posicionado no gargalo do frasco para condensar os vapores. Após resfriada, a solução foi centrifugada por $15 \mathrm{~min}$ a $1500 \mathrm{rpm}$, descartando o sobrenadante. Ao precipitado foi adicionado $50 \mathrm{~mL}$ de álcool etílico $95 \%$ e novamente centrifugado por $10 \mathrm{~min}$ a $1500 \mathrm{rpm}$. O precipitado foi novamente lavado com $500 \mathrm{~mL}$ de álcool $70 \%$ e centrifugado nas mesmas condições anteriores.

Ao precipitado, foram adicionados $150 \mathrm{~mL}$ de água destilada e 5 gotas da solução de hidróxido de sódio $(\mathrm{NaOH})$ a $10 \%$, sendo, posteriormente, autoclavado a $100{ }^{\circ} \mathrm{C}$ por $1 \mathrm{~h}$. Após resfriamento, adicionou-se $5 \mathrm{~mL}$ de ácido clorídrico $(\mathrm{HCl})$, aquecido novamente em autoclave durante 30 minutos e neutralizado com uma solução de hidróxido de sódio a $10 \%$. Transferiu-se a solução para balão volumétrico de $500 \mathrm{~mL}$, completouse o volume com água e filtrou-se em papel filtro. $\mathrm{O}$ filtrado foi usado para determinação de açúcares redutores, empregando o método de DNS (ácido 3,5dinitrosalicílico), conforme descrito no método 038/IV do Instituto Adolfo Lutz (2008).

\section{Análise estatística}

Todas as análises foram realizadas em triplicata, submetidas ao teste de Tukey com nível de significância de $5 \%$ e analisadas através do software Statistic versão 7.0 (Calado e Montgomery, 2003).

\section{Resultados e Discussão}

\section{Avaliação físico-química da fruta-pão in natura e desidratada (farinha)}

As análises físico-químicas de umidade, cinzas, acidez e $\mathrm{pH}$ foram realizadas em todas as partes da fruta in natura, porém, na farinha, não foi possível realizar as análises do caroço, devido à baixa quantidade de amostra, uma vez que o mesmo se encontra em menor quantidade na fruta. A análise do amido foi realizada somente na amostra seca (farinha), uma vez que se acredita que a fruta apresenta potencial para ser aplicada na forma de farinha.

Há poucos relatos na literatura sobre a caracterização físico-química da fruta in natura e da farinha da fruta-pão de sementes, o que evidencia ainda mais a importância dos resultados obtidos neste trabalho. A Tabela 2 apresenta as análises físico- químicas de umidade, cinzas, $\mathrm{pH}$ e acidez em ácido cítrico para as diferentes partes da fruta pão in natura e desidratada (farinha).

A fruta-pão pode ser considerada uma fruta com elevada umidade, sendo que o caroço foi a parte que apresentou maior umidade e, a semente, a menor na fruta in natura. Para a farinha, como era esperado, o teor de umidade foi reduzido devido à evaporação de água no processo de secagem, sendo que a semente também apresentou a menor umidade dentre as farinhas avaliadas.

Segundo Portaria no 354, de 18 de julho de 1996 da Agência Nacional de Vigilância Sanitária (ANVISA) (Brasil, 1996), o valor máximo de umidade para farinhas é de $15 \%$. Assim, apenas a farinha da polpa não atende aos parâmetros legislados. No entanto, a parte dessa fruta mais utilizada é a semente, pelo fato de apresentar em torno de $13 \%$ de proteínas (Falcão et al., 2001).

De acordo com a Tabela Brasileira de Composição de Alimentos (Taco, 2011), a umidade da fruta-pão é de $80,90 \%$, valor este semelhante ao obtido na casca e semente. Souza e Souza (2012), ao avaliarem a frutapão da variedade Apyrena, encontraram valores de umidade para fruta in natura correspondente a $74,58 \pm 2,84 \%$, valor abaixo do apresentado no presente estudo. Porém, é importante ressaltar que além das frutas serem de variedades diferentes, fatores como regiões, grau de maturação, características do solo, clima e época de colheita interferem nas características físico-químicas de frutas (Morzelle et al., 2015).

Assim como a umidade, o caroço apresentou o maior conteúdo de cinzas, seguido pela polpa, casca e semente. Na farinha, como era esperado, o teor de cinzas aumentou devido à perda de água na secagem e consequente concentração dos nutrientes nas diferentes partes da fruta. Comparando com os resultados obtidos por Santos et al. (2012) em relação à farinha obtida a partir do caroço da jaca (Artocarpos integrifólia L.), eles obtiveram um teor de cinzas de 2,57 \%. Em contraste, Pereira et al. (2010) avaliou a fruta-pão de massa e conseguiu um teor de $4,60 \pm 0,44 \%$ de cinzas; para a farinha da polpa, ambos os valores são inferiores aos obtidos no presente trabalho.

A semente é parte do fruto in natura, que se destaca por conter o maior $\mathrm{pH}(5,55 \pm 0,05)$. As outras partes não apresentaram diferença entre si, sendo que a polpa

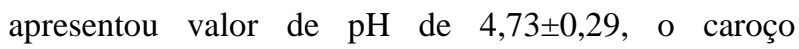
$4,50 \pm 0,10$ e a casca $4,38 \pm 0,02$. Quando comparada à farinha com amostra in natura, observa-se que, das partes avaliadas, apenas a polpa apresentou variação do pH após a secagem. Souza e Souza (2012) avaliaram o $\mathrm{pH}$ da polpa da variedade Apyrena e encontraram valores de $\mathrm{pH}$ para a amostra in natura $(6,01 \pm 0,04) \mathrm{e}$ para a farinha $(5,55 \pm 0,02)$. 
Tabela 2. Valores de umidade, cinzas, pH e acidez das diferentes partes que compõem a fruta-pão (casca, polpa, semente e caroço).

\begin{tabular}{|c|c|c|c|}
\hline Parâmetros & Parte da fruta & in natura & Farinha \\
\hline \multirow{5}{*}{ Umidade (\%) } & Casca & $80 \pm 0,45^{\mathrm{c}}$ & $12,95 \pm 0,42^{\mathrm{b}}$ \\
\hline & Polpa & $85 \pm 0,15^{\mathrm{b}}$ & $16,18 \pm 0,10^{\mathrm{a}}$ \\
\hline & Semente & $79 \pm 0,39^{d}$ & $9,98 \pm 0,66^{c}$ \\
\hline & Caroço & $89 \pm 0,10^{\mathrm{a}}$ & - \\
\hline & $\mathrm{CV}(\%)$ & 5,58 & 13,04 \\
\hline \multirow{5}{*}{ Cinzas (\%) } & Casca & $7,85 \pm 0,31^{\mathrm{b}}$ & $8,72 \pm 0,61^{b}$ \\
\hline & Polpa & $7,93 \pm 0,40^{\mathrm{b}}$ & $9,19 \pm 0,36^{\mathrm{a}}$ \\
\hline & Semente & $4,23 \pm 0,05^{\mathrm{c}}$ & $6,64 \pm 0,18^{c}$ \\
\hline & Caroço & $9,33 \pm 0,27^{\mathrm{a}}$ & - \\
\hline & CV (\%) & 29,70 & 16,58 \\
\hline \multirow{5}{*}{$\mathrm{pH}$} & Casca & $4,38 \pm 0,02^{b}$ & $4,38 \pm 0,018^{b}$ \\
\hline & Polpa & $4,73 \pm 0,29^{b}$ & $4,02 \pm 0,25^{\mathrm{c}}$ \\
\hline & Semente & $5,55 \pm 0,05^{\mathrm{a}}$ & $5,50 \pm 0,23^{\mathrm{a}}$ \\
\hline & Caroço & $4,50 \pm 0,10^{\mathrm{b}}$ & - \\
\hline & CV (\%) & 11 & 16,66 \\
\hline \multirow{5}{*}{ Acidez (\%) } & Casca & $0,06 \pm 0,0002^{\mathrm{d}}$ & $0,176 \pm 0,003^{b}$ \\
\hline & Polpa & $0,07 \pm 0,0001^{\mathrm{c}}$ & $0,460 \pm 0,004^{a}$ \\
\hline & Semente & $0,045 \pm 0,0001^{\mathrm{b}}$ & $0,129 \pm 0,005^{\mathrm{c}}$ \\
\hline & Caroço & $0,048 \pm 0,0007^{\mathrm{a}}$ & - \\
\hline & $\mathrm{CV}(\%)$ & 20,63 & 25,13 \\
\hline
\end{tabular}

*Médias seguidas pela mesma letra na coluna não diferem entre si para cada uma das análises, pelo teste de Tukey $(\mathrm{p}<0,05)$.

$\mathrm{Na}$ comparação com outras frutas, Canuto et al. (2010) encontraram pH de 3,30 para a polpa de açaí (Euterpe oleracea), $\mathrm{pH}$ de 2,80 para acerola (Malpighia emarginata), pH de 4,70 para caju (Anacardium occidentale L.) e $\mathrm{pH}$ de 3,50 para o cupuaçu (Theobroma grandiflorum). Aragão et al. (2017) encontraram o pH de 3,67 para a manga (Mangifera indica) e Carlos et al. (2017) avaliaram diversas partes do fruto murici (Byrsonima crassifolia) (casca, polpa e semente), encontrando $\mathrm{pH}$ que variou de 3,5 a 4,3.

As análises realizadas com as partes da fruta-pão indicaram que as partes da fruta in natura apresentaram uma acidez menor se comparadas com as partes como farinha. Estes resultados estão de acordo com o pH, uma vez que a polpa apresentou menor $\mathrm{pH}$ e, a semente, o valor mais elevado. Souza e Souza (2012), em seus estudos com a fruta-pão in natura da variedade Apyrena, observaram em seus resultados valores para acidez titulável equivalente a $1,64 \pm 0,01 \%$, e não detectável para as amostras secas (farinha).

$\mathrm{Na}$ análise do amido na fruta-pão de semente, a parte em que mais foi encontrado teor de amido foi na semente, correspondendo a $38,00 \%$, seguida da polpa $(19,10 \%)$, caroço e casca com $15,94 \%$, não diferenciando entre si, como mostra a Figura 1.

Pesquisas realizadas por Santos et al. (2013) indicaram que o teor de amido para as sementes de jaca mole e jaca dura foram de $31,73 \%$ e $30,93 \%$, respectivamente. Já Luciano et al. (2017) encontraram para a jaca mole $29,1 \pm 0,1 \%$ e, para a jaca dura, $24,1 \pm 0,1 \%$, utilizando água como solvente para a extração do amido; e utilizando $\mathrm{NaOH}$ como solvente, obtiveram para jaca mole $32,1 \pm 0,05 \%$ e, para jaca dura, $26,5 \pm 0,04 \%$.

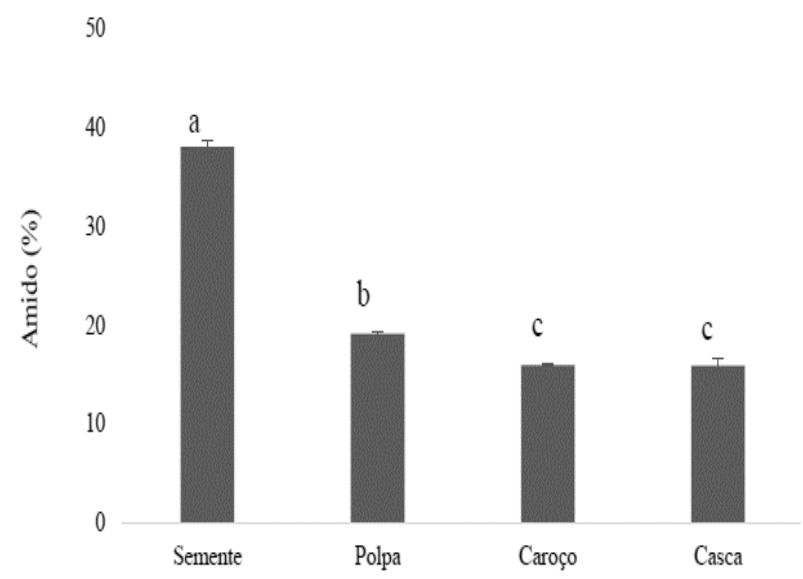

Figura 1. Teor de amido da amostra desidratada (farinha) das diferentes partes da fruta-pão (semente, polpa, caroço e semente). *Médias seguidas pela mesma letra na coluna não diferem entre si pelo teste de Tukey $(\mathrm{p}<0,05)$

Em estudos realizados por Silva et al. (2017) com a banana maçã (Musa acuminata), verificou-se que os teores de amido sofrem influência tanto do estado de maturação quanto entre as amostras in natura $\mathrm{e}$ 
desidratadas, sendo que, quando desidratadas, as amostras apresentaram o triplo de amido em comparação com a fruta in natura, em todos os estados de maturação (verde-73,02 $\pm 2,39 \%$; madura-

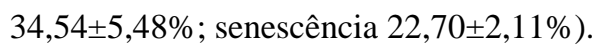

Esses resultados evidenciam que a fruta-pão de semente tem potencial para ser explorada como fonte de amido em formulações alimentícias, uma vez que os teores de amido obtidos no presente estudo não diferenciam expressivamente quando comparados com outras frutas descritas na literatura.

\section{Curvas de secagem}

No decorrer da secagem, as amostras foram pesadas constantemente até atingirem peso constante. Diversos modelos matemáticos têm sido utilizados para descrever o processo de secagem de produtos agrícolas. Embora várias teorias tenham sido propostas para predizer o comportamento da secagem de grãos e sementes, na maioria das vezes, as relações semi-empíricas e empíricas têm-se mostrado como melhores opções para predizer o processo de secagem (Resende et al., 2010). No presente trabalho, as curvas foram tratadas usando três modelos matemáticos amplamente empregados na secagem de matérias-primas agroindustriais: Henderson e Pabis, Midilli e Kucuk, e Page. Os parâmetros destes modelos matemáticos estão representados na Tabela 3, assim como a correlação e o erro obtidos em cada modelagem.

Ao observar a Tabela 3, percebe-se que, em função da correlação dos três modelos, os que têm os menores valores para as quatro partes da fruta-pão são os de
Henderson e Pabis, indicando assim um menor ajuste desse modelo aos dados experimentais. Quando se observa os modelos de Page, Midilli e Kucuk percebese que as suas correlações estão bem similares entre si. Além da correlação, estes dois últimos modelos também apresentam valores de erro médio padrão próximos. Porém, o modelo de Midilli e Kucuk possui quatro parâmetros, enquanto que o modelo de Page tem apenas dois. Assim, baseado nos parâmetros estatísticos, a equação de Page foi selecionada como o modelo que melhor se ajusta aos dados experimentais.

Outros estudos reportados na literatura também apontam a equação de Page como o melhor modelo para ajuste dos dados experimentais. Santos et al. (2010), ao submeter seus dados de secagem de carambola aos modelos de Henderson e Pabis, Exponencial e Page, pôde constatar que o modelo de Page apresentou o maior valor de correlação e um menor erro médio relativo. Alexandre et al. (2009), em seus estudos sobre a cinética de secagem do abacaxi em fatias, submeteram a fruta à secagem em três temperaturas e observaram que o modelo de Page foi o que apresentou os melhores ajustes com maiores valores para a correlação. Menezes et al. (2013), ao analisar a cinética e os ajustes dos modelos matemáticos aos dados encontrados na secagem de bagaço de maracujá-amarelo, concluíram que, dos modelos empregados, o que melhor se ajustou aos dados experimentais foi o de Page, que apresentou a maior correlação e menores erros. Silva et al. (2017) também verificaram que o modelo de Page foi o que melhor se ajustou à cinética de secagem da biomassa da banana maçã.

Tabela 3. Dados do ajuste das curvas de secagem das diferentes partes da fruta-pão de semente secas a $40{ }^{\circ} \mathrm{C}$ nos três modelos matemáticos aplicados.

\begin{tabular}{cccccc}
\hline Modelo & Parâmetro & Casca & Polpa & Caroço & Semente \\
\hline Henderson e Pabis & $\mathrm{a}$ & 1,300200 & 1,000100 & 1,090910 & 1,000093 \\
& $\mathrm{k}$ & 0,021113 & 0,021114 & 0,012227 & 0,019000 \\
& Correlação & 0,940107 & 0,965151 & 0,946216 & 0,947689 \\
Midilli e Kucuk & Erro & 4,918529 & 4,467774 & 4,744488 & 4,138569 \\
& $\mathrm{a}$ & 0,900000 & 1,006275 & 1,009999 & 1,006275 \\
& $\mathrm{~b}$ & 0,000011 & 0,000002 & 0,000000 & 0,000002 \\
& $\mathrm{k}$ & 0,000235 & 0,000514 & 0,000360 & 0,000514 \\
& $\mathrm{n}$ & 1,899999 & 1,849153 & 1,849101 & 1,849153 \\
Page & Correlação & 0,994693 & 0,998416 & 0,983026 & 0,988739 \\
& Erro & 0,608646 & 0,208619 & 0,732416 & 0,380508 \\
& $\mathrm{k}$ & 0,000200 & 0,000222 & 0,000169 & 0,000220 \\
& $\mathrm{n}$ & 2,000000 & 2,050000 & 2,009999 & 2,050000 \\
& Correlação & 0,998057 & 0,999281 & 0,988078 & 0,992358
\end{tabular}


A qualidade dos ajustes também pode ser observada nas curvas de secagem dos modelos matemáticos, que estão apresentadas na Figura 2, que mostra o comportamento cinético das amostras das diferentes partes da fruta-pão de sementes, submetidas à secagem na temperatura de $40{ }^{\circ} \mathrm{C}$.

$\mathrm{Na}$ Figura 2, é possível observar que a curva da semente (D) foi a que obteve primeiro seu equilíbrio, necessitando em torno de $120 \mathrm{~min}$, em razão de ser a parte da fruta que obteve menos umidade, seguida da polpa (B) e do caroço (C), que obtiveram seu equilíbrio com 150 min e, por último, a casca (A), devido à forma gastando mais que $150 \mathrm{~min}$ para atingir seu equilíbrio. Observa-se também que o modelo que menos se ajustou à curva foi o de Henderson e Pabis, e o melhor modelo observado foi o de Page, sendo que foi na secagem da casca em que ele melhor se ajustou visualmente.
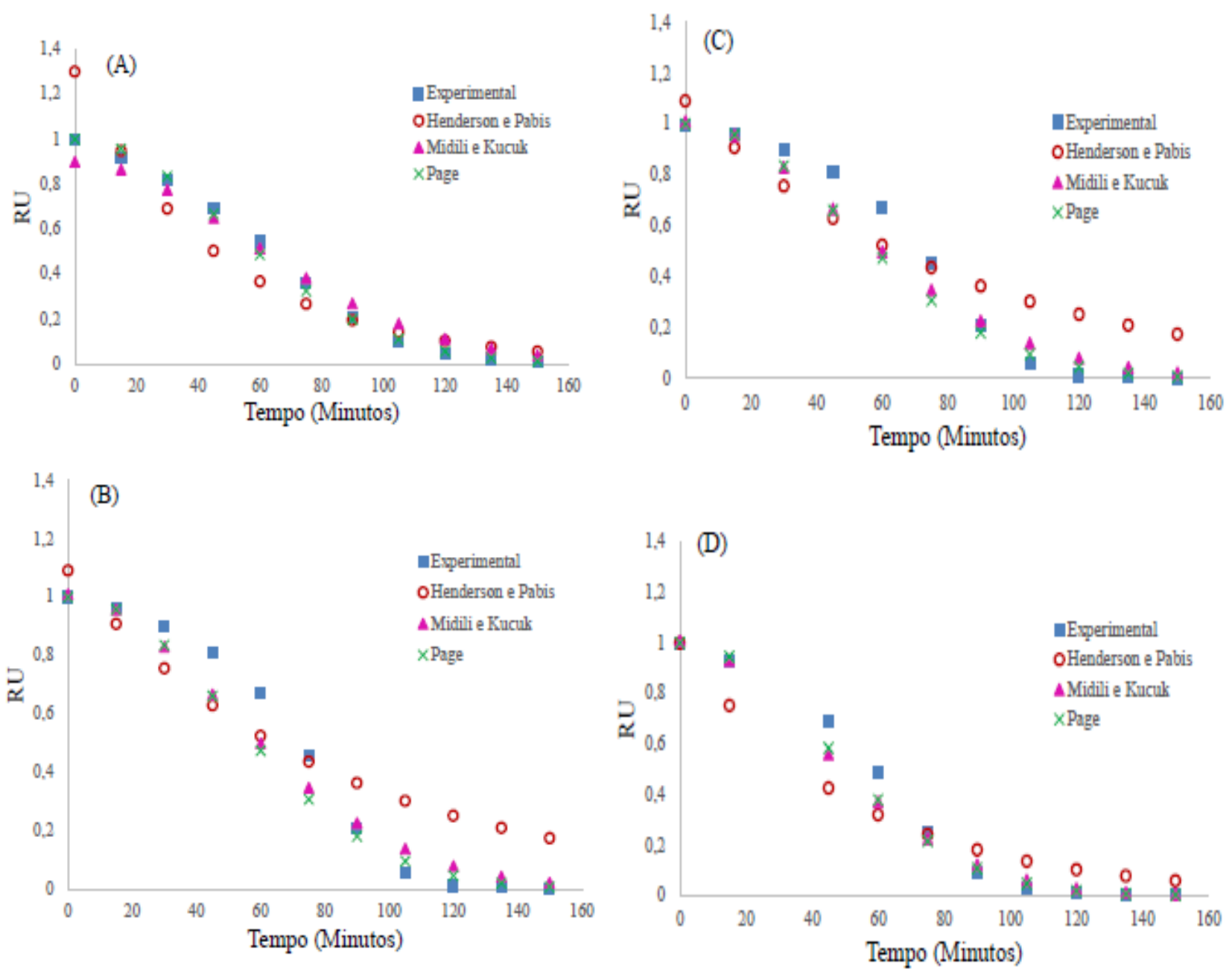

Figura 2. Curvas de secagem das partes da fruta-pão empregando os modelos matemáticos: Casca (A), Polpa (B), Caroço (C), Semente (D).

\section{Conclusões}

A caracterização da casca, polpa, semente e caroço da fruta-pão de semente mostra que a fruta apresenta elevados teores de umidade e cinzas e $\mathrm{pH}$ baixo em todas as suas partes. A fruta-pão de sementes apresentou um percentual de amido considerável, podendo assim, com estudos mais profundos, ser utilizada em novas formulações de alimentos. Com relação à curva de secagem, o modelo de Page foi o que melhor se ajustou aos dados experimentais para todas as partes da frutapão de semente.

\section{Agradecimentos}

Os autores agradecem à Universidade do Estado de Mato Grosso (UNEMAT), pelo apoio estrutural, à Fundação de Amparo à Pesquisa do Estado de Mato Grosso (FAPEMAT), pelo apoio financeiro (processo $\left.\mathrm{n}^{\circ} 0214457 / 2018\right)$ e ao Conselho Nacional de Desenvolvimento Científico e Tecnológico (CNPq), pela bolsa de pesquisa DCR (processo n`313859/20175). 


\section{Referências Bibliográficas}

Almeida, E.J., Jesus, N., Scaloppi, E.M.T., Martins, A.B.G., Araújo, M.S., 2008. Propagação de três genótipos de abieiro (Pouteria caimito) por estaquia de ramos herbáceos. Acta Amazônica, 38, 1-4.

Alexandre, H.V., Gomes, J.P., Barros Neto, A.L., Silva, F.L.H., Almeida, F.A.C., 2009. Cinética de secagem de abacaxi pérola em fatias. Revista Brasileira de Produtos Agroindustriais, 11, 123-128.

Aragão, P.P., Loss, R.A., Silva, S.S., Guedes, S.F., 2017. Avaliação do potencial de utilização de pré-tratamento osmótico na produção de manga desidratada. Revista Destaques Acadêmicos, 9, 161- 173.

Brasil. Agência Nacional de Vigilância Sanitária (1996). Institui o Regulamento Técnico sobre a identidade e as características mínimas de qualidade a que deverá obedecer a farinha de trigo. Portaria $\mathrm{n}^{\circ} 354$, de 18 de julho de 1996. Diário Oficial da República Federativa do Brasil.

Calado, V., Montgomery, D., 2003. Planejamento de experimentos usando Statistica. E-papers serviços editoriais, Rio de Janeiro.

Canuto, G.A.B., Xavier, A.A.O., Neves, L.C., Benassi, M.T., 2010. Caracterização físico-química de polpas de frutos da amazônia e sua correlação com a atividade anti-radical livre. Revista Brasileira de Fruticultura, 32, 1196-1205.

Carlos, N.D.A., Loss, R.A., Silva, S.S., Guedes, S.F., Carvalho, J.W.P., 2017. Avaliação físico-química e atividade antimicrobiana da casca, polpa e semente do murici (Byrsonima crassifolia). Enciclopédia Biosfera, 14, 232- 243.

Celestino, S.M.C., 2010. Princípios de secagem de alimentos. Embrapa Cerrados, Planaltina.

Falcão, M.A., Clement, C.R., Gomes, J.B.M., Flores, W.B.C., Santiago, F.F., Freitas, V.P., 2001. Fenologia e produtividade da fruta-pão (Artocarpus altilis) e da jaca (A. hetephyllus) na Amazônia Central. Acta amazônica, 31, 179-191.

Farias, L.L., Loss, R.A., Silva, S.S., Puton, B.M.S., Paroul, N., Cansian, R.L., Guedes, S.F., 2018. Potencial antimicrobiano e avaliação físico-química da casca, polpa e biomassa do noni (Morinda citrifolia Linn). Global Science and Technology, 11, 247-255.

Instituto Adolfo Lutz, 2008. Métodos físico-químicos para análise de alimentos, fourth ed. Instituto Adolfo Lutz, Brasília.

Luciano, C.G., Valencia, G.A., Campanella, O.H., Sobral, P.J.A., Moraes, I.C.F., 2018. Influence of extraction method on the rheological properties of jackfruit (Artocarpus heterophyllus) seed starch dispersions. Food Biophysics, 13, 155-162.

Luciano, C.G., Franco, C.M.L., Valencia, G.A., Sobral, P.J.A., Moraes, I.C.F., 2017. Evaluation of extraction method on the structure and physicochemical properties of starch from seeds of two jackfruit varieties. Starch-Stärke, 69, 11-12.

Madruga, M.S., Albuquerque, F.S.M., Silva, I.R.A., Amaral, D.S., Magnani, M., Queiroga Neto, V., 2014. Chemical, morphological and functional properties of Brazilian jackfruit (Artocarpus heterophyllus L.) seeds starch. Food Chemistry, 143, 440- 445.
Melo, K.S., Figueirêdo, R.M.F., Queiroz, A.J.M., Fernandes, T.K.S., Bezerra, M.C.T., 2013. Secagem em camada de espuma da polpa do fruto do mandacaru: experimentação e ajustes de modelos matemáticos. Revista Caatinga, 26, 10-17.

Menezes, M.L., Straher, A.P., Pereira, N. C., Barros, S.T.D., 2013. Análise da cinética e ajustes de modelos matemáticos aos dados de secagem do bagaço do maracujá-amarelo. Revista da Escola de Engenharia da UFF, 15, 176-186.

Morzelle, M.C., Bachiega, P., Souza, E.C., Vilas Boas, E.V. B., Lamounier, M.L., 2015. Caracterização química e física de frutos de curriola, gabiroba e murici provenientes do cerrado brasileiro. Revista Brasileira de Fruticultura, 37, 96- 103.

Pereira, S.C.L., Monteiro, M.R.P., Henriques, G.S., Pavesi, M.M., Amorim, F.N., 2010. Análise do efeito prebiótico da farinha de fruta-pão (Artocarpus incisa) sobre a microbiota intestinal de ratos Wistar. Revista do Médico Residente, 12, 111 .

Pradhan, C., Mohanty, M., Rout, A., 2012. Phytochemical screening and comparative bioefficacy assessment of Artocarpus altilis leaf extracts for antimicrobial activity. Frontiers in Life Science, 6, 71-76.

Resende, O., Ferreira, L.U., Almeida, D.P., 2010. Modelagem matemática para descrição da cinética de secagem do feijão adzuki (Vigna angularis). Revista Brasileira de Produtos Agroindustriais, 12, 171-178.

Santos, L.S., Bronomo, R.C.F., Fontam, R.C.I., Bronomo, P., Leite, C.X.S., Santos, D.O., 2013. Efeito dos métodos de extração na composição, rendimento e propriedades da pasta do amido obtido da semente de jaca. Revista Brasileira de Produtos Agroindustriais, 15, 255-261.

Santos, C.T., Bonomo, R.F., Chaves, M.A., Fontan, R.C.I., Bonomo, P., 2010. Cinética e modelagem da secagem de carambola (Averrhoa carambola L.) em secador de bandeja. Acta Scientiarum Technology, 32, 309-313.

Santos, D.B., Machado, M.S., Araújo, A.F., Cardoso, R.L., Tavares, J.T.Q., 2012. Desenvolvimento de pão francês com a adição de farinha de caroço de jaca (Artocarpos integrifólia L.). Enciclopédia Biosfera, 8, 597-602.

Sacramento, C.K., Leite, J.B.V., Carvalho, J.E.U., Nascimento, W.M.O., 2009. Fruta-pão, in: Santos-Serejo, J. A., Dantas, J.L.L., Sampaio, C.V., Coelho, Y.S., (Ed.), Fruticultura tropical: espécies regionais e exóticas. Embrapa Informação Tecnológica, Brasília, pp. 185-200.

Silva, K.C., Neris, T.S., Loss, R.A., Silva, S.S., Guedes, S.F., 2017. Caracterização da biomassa da banana-maçã (musa spp.) em diferentes estádios de maturação in natura e desidratada. Enciclopédia Biosfera, 14, 131- 143.

Souza, D.S., Souza, J.D.R.P., 2012. Elaboração de farinha instantânea a partir da polpa de fruta-pão (Artocarpus altilis). Ciência Rural, 42, 1123 - 1129.

Taco. Tabela Brasileira de Composição de Alimentos, 2011. four ed. NEPA- UNICAMP, Campinas.

Virgolin, L.B., Seixas, F.R.F., Janzantti, N.S., 2017. Composition, content of bioactive compounds, and antioxidant activity of fruit pulps from the Brazilian Amazon biome. Pesquisa Agropecuária Brasileira, 52, 933-941. 